\title{
Target Detection in SAR Images Based on a Level Set Approach
}

\author{
Regis C. P. Marques, Fátima N. S. Medeiros and Daniela M. Ushizima
}

March 20, 2009

\begin{abstract}
This paper introduces a new framework for target detection in SAR images. We focus on the task of locating heterogeneous regions using a level set based algorithm. Unlike most of the approaches in image segmentation, we address an algorithm which incorporates speckle statistics instead of empirical parameters and discards speckle filtering. The curve evolves according to speckle statistics, initially propagating with a maximum upward velocity in homogeneous areas. Our approach is validated by a series of tests on synthetic and real SAR images demonstrating that it represents a novel and efficient method for target detection purpose.
\end{abstract}

\section{Introduction}

Image segmentation is a challenging issue in digital image processing. Several applications in shape analysis and pattern recognition require robust

*Manuscript under revision by January 28th, 2008.

${ }^{\dagger}$ This is not a final version - final version available on IEEE Trans Systems, Man and Cybernetics - part C, vol.39, n.2, pp.214:222, 2009.

${ }_{\ddagger}^{\ddagger}$ R. C. P. Marques is with Federal Center of Education - CEFET, Fortaleza, CE, Brazil.

${ }^{\S}$ F. N. S. Medeiros is with Teleinformatics Department, Federal University of Ceara DETI/UFC, Fortaleza, CE, Brazil.

ID. M. Ushizima is with Math and Visualization Groups, Lawrence Berkeley National Laboratory - LNBL, Berkeley, CA, USA. Work supported by the U.S. Department of Energy under Contract No. DE-AC02-05CH11231. 
segmentation methods, which have clear implications on the success of posterior analysis. An important application is target detection in SAR (Synthetic Aperture Radar) images, which has motivated several publications as in $[1,2,3,4,5,6]$.

SAR imagery systems are based on the detection of reflected microwaves emitted by a satellite, resulting on images degraded by speckle noise. Speckle noise reduction is necessary to detect targets, but small targets can be eliminated by the filtering process due to its typical limitations in preserving sharp features and details of the original image [7].

In order to overcome these effects, filtering-free techniques have been proposed to SAR image processing [8, 9, 10]. Detection of targets in SAR signals regard object segmentation and level sets have improved traditional filteringsegmentation approaches such the one proposed by [11], which regards region snakes using the maximum likelihood method for different noise models for image segmentation.

The advantage of using front propagations is the ability to deal with segmentation of objects lacking sharp edges [12]. Such techniques have been applied mainly in optical images [13, 14, 15, 16, 17], but Huang et. al [2] developed a level set formulation for oil slick segmentation in SAR images. This method integrates models of intensity and curvature to determine the intensity gradient-driven and curvature driven front propagation speed. Their results presented image segmentations without information loss, an advantage of this method, despite speckle noise removal during the front propagation. Differently from this work, our framework includes speckle statistics over local regions in the front propagation model to enclose targets and fine details in $L$-looks amplitude SAR images.

A similar approach, proposed in [18] for intensity SAR image segmentation, addressed the segmentation by dividing the image into a given but arbitrary number $N$ of Gamma-homogeneous regions. The active contours evolution was achieved via level sets. The algorithm consisted in evolving closed planar curves within an explicit correspondence between the interior of the curves and the regions to be segmented in order to minimize a criterion based on the speckle noise model and a regularization term. The ability of the algorithm to handle automatically topological changes of curves provided regions of disjoint components to be addressed during the segmentation process. This statistical framework succeeded in locating the boundaries of the predefined homogeneous regions, with the disadvantage it required an initial partition $(N)$ of the image. 
Our paper is motivated by the numerical efficiency accomplished when discarding SAR preprocessing or postprocessing stages, with reduced noise sensitivity to the segmentation method. This paper presents a level set evolution model, addressing the movement of the front according to speckle statistics. The front moves outward faster in homogeneous regions (e.g. background) than in heterogeneous regions (e.g. targets), incorporating image statistics and a multiscale approach for differentiating the intensity variations among the regions. This model incorporates a local neighborhood homogeneity measure based on speckle statistics [19] and an adaptive windowing scheme [20] to achieve target detection in speckled images.

This paper is organized as follows: Section 2 defines terms, SAR image and its modeling as well as SAR segmentation methods to be compared with about the speckle noise model and level set technique. The proposed propagation speed model is introduced in Section 3. Section 4 presents the simulation results. Conclusions and further improvements are summarized in Section 5.

\section{Background}

SAR images are the result of a sophisticated processing of radar data, captured from the reflection of emitted beams, subjected to the scattering properties of the materials that lie in the path of the microwave beam. SAR systems can process the return signal in amplitude or intensity modes of single or multi-look data to find targets. Target detection is achieved by narrowing the concept of texture, concerning with variations that are somehow associated with the "roughness" of the surface that describes the backscatter as a function of space in a radar image. These textures can be generated due to reflectivity changes on terrain, which indicates a target or background. Depending on the homogeneity of the target, i.e homogeneous, heterogeneous or extremely heterogeneous, different distributions will be used to model this target [1].

In this paper, we address amplitude images and inherent speckle noise using multi-look data as mean of detecting targets. Statistically, the surface is considered to be a random process in 2D and analyzed using local estimates (variance) and global variations of the image, combining the proposed speed function and curvature. For additional information about SAR image acquisition and target detection based on texture segmentation, the reader 
is referred to $[21,22,23]$.

\subsection{The Speckle noise multiplicative model}

Speckle noise $(Z)$ is often modeled as a multiplicative noise over a noise-free image $X$, where $Z$ is supposed to be uncorrelated to $X$ [24]. The model follows the expression:

$$
Y=X . Z
$$

where $Z$ has an unitary mean with variance $\sigma_{z}^{2}$. As $Z$ and $X$ are assumed to be statistically independent, the sample mean and the variance over homogeneous areas, i.e. areas of constant average intensity, can be derived from the expressions [19]:

$$
\begin{gathered}
\bar{Y}=\bar{X} \cdot \bar{Z}=\bar{X} \\
\sigma_{z}=\frac{\sigma_{y}}{\bar{X}}
\end{gathered}
$$

The speckle noise standard deviation $\left(\sigma_{z}\right)$ provides the amount of variation of terrain reflectivity in SAR images for regions where the multiplicative model holds [24]. Speckle presents a well known statistical model for homogeneous regions [20], which can be quantified using Eq. 4.

$$
\beta_{z}=\frac{0.5227}{\sqrt{L}}
$$

The variation coefficient $\left(\beta_{z}\right)$ represents the speckle noise fluctuations in SAR images, calculated according to Eq.4, which can be used as a measure of homogeneity. For homogeneous regions, $\sigma_{z}=\beta_{z}$ and the sample mean, $\left(\mu_{y}\right)$, and standard deviation, $\left(\sigma_{y}\right)$ can be estimated over a window comprising $W$ pixels in these homogeneous regions as follows:

$$
\begin{gathered}
\hat{\mu}_{y}=\bar{Y}=\bar{X}=\frac{1}{W} \sum_{i=1}^{W} Y_{i} \\
\hat{\sigma}_{y}=\sqrt{\frac{\sum_{i=1}^{W}\left(Y_{i}-\hat{\mu}_{y}\right)^{2}}{W-1}}
\end{gathered}
$$


where $Y_{i}$ denotes the pixel value.

\subsection{Speckle statistics for multilook amplitude data}

Considering speckle statistics for amplitude images, which follows the square root of gamma distribution, we can state that Eq. 3 and Eq. 4 are true only for homogeneous regions of amplitude images, given any $L$ [19].

The hypothesis that governs the modeling of homogeneous regions in SAR images is that the backscatter is constant, though its value is unknown and for inhomogeneous ones the backscatter is not constant [25].

Multilook amplitude speckle is calculated from the square root of the multilook intensity speckle, then Eq. 7 presents the square root of a gamma distribution [26].

$$
P_{L}(A)=\frac{2 L^{L}}{\left(\sigma^{2}\right)^{L} \Gamma(L)} A^{2 L-1} \exp \left(-\frac{\left(L A^{2}\right)}{\sigma^{2}}\right)
$$

for $L \geq 1$ and $A>0$, where $A$ is the amplitude noise signal and $\Gamma(\cdot)$ is the gamma function. When $L$ is equal to one (i.e. single-look amplitude) the speckle is modeled by a Rayleigh distribution [22].

In case that the number of looks $(L)$ is unknown, it can be estimated over those areas where the multiplicative model holds. The estimator of $L$, denoted by $\hat{L}$ has been referred to as the "equivalent number of looks" in $[27,28,1]$. By using the moments method the estimator is the solution of the following equation [28]

$$
\left(\sqrt{\frac{\hat{m}_{2}}{\hat{L}}}\right) \frac{\Gamma\left(\hat{L}+\frac{1}{2}\right)}{\Gamma(\hat{L})}-\hat{m}_{1}=0
$$

where $\hat{m}_{j}$ denotes the jth-order sample moment.

The backscatter (or clutter) that describes the ground truth $(X)$ may exhibit different degrees of homogeneity, and different models can be used to encompass peculiarities. Three main models have proved useful in modeling amplitude backscatter: a constant whenever the area is homogeneous to the sensor, the square root of gamma distributed random variable for heterogeneous areas and, more recently, the square root of the reciprocal of a gamma distributed random variable, for extremely heterogeneous areas 
[29]. These three situations are unified by the square root of the generalized inverse Gaussian distribution and are adequate to model real data [29, 26].

Elementary tasks in SAR image processing such as segmentation, edge or target detection become difficult due to the speckle noise. Preprocessing steps as speckle filtering are not recommended since they cause loss of relevant information. The contribution of the current paper relies on a level set-based algorithm to perform target detection without requiring neither preprocessing nor postprocessing. Furthermore, it incorporates speckle statistical properties in the front-driven speed showing the feasibility of the level set algorithm to evolve to target boundaries in speckled images.

Next sections introduce the methods to be compared with the proposed algorithm.

\subsection{Thresholding and ICM}

SAR imagery analysis has been addressed using several algorithms as thresholding and iterated conditional modes (ICM) [4, 30, 31, 3]. Although thresholding methods are one of the oldest approaches in image segmentation, they are simple, fast and still render practical results [32, 30].

The thresholding method computed for this paper consider an entropic algorithm, where the threshold is estimated according to entropy measurements over the image histogram [31].

The ICM algorithm is an iterative method based upon the modeling of the a priori distribution for the classes with a multiclass Potts-Strauss Markov random field (MRF) framework [3]. It is a quick deterministic optimization method for MRF, which gives a good sub-optimal solution in a few (in linear time) steps, if a suitable initial labeling is available a priori.

\subsection{The level set method}

Applications of propagating fronts using level set formulation to image segmentation have been reported successfully in recent publications $[11,2,18$, 33], particularly when incorporating priori scene knowledge to the algorithms. The efficiency and robustness of such methods motivated the current paper, where we combine level set methods and speckle noise statistics to detect targets in noisy images.

The central idea in the level set approach is to represent a front $\gamma(t)$ as the initial level $\psi(\mathbf{x}, t)=0$ of a surface $\psi(\mathbf{x}, \mathbf{t})$, where $\mathbf{x} \in \Re^{n}$ [34]. The goal 
is to produce a front motion as consequence of surface motion, in which $\gamma(t)$ is embedded in accordance to:

$$
\gamma(t)_{t=0}=(\mathbf{x} \mid \psi(\mathbf{x}, t)=0)_{t=0} .
$$

In Sethian [34], the rate of surface motion is defined in terms of $\partial \psi / \partial t=$ $F|\nabla \psi|$. Thus, $|\nabla \psi|$ indicates the difference operator and $F$ is a scalar function that defines the speed in the upward direction normal to $\psi$. The surface evolution equation is straightforward, namely

$$
\psi_{n+1}=\psi_{n}+\Delta t \cdot F\left|\nabla \psi_{n}\right|
$$

Fig.1 exhibits a level set evolution example. Fig. 1(a) shows the initial front $\gamma(t=0)$ from which the level set zero $(x, t=0)$ is computed and Fig.1(b) illustrates this process. Using Eq. 10 and the speed model described in Eq. 11 the front goes to a quite distinct surface $\psi$ (Fig.1(c)). Fig. 1(d) represents the updated front following Eq.9.

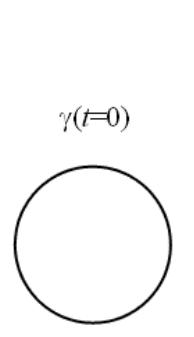

(a)

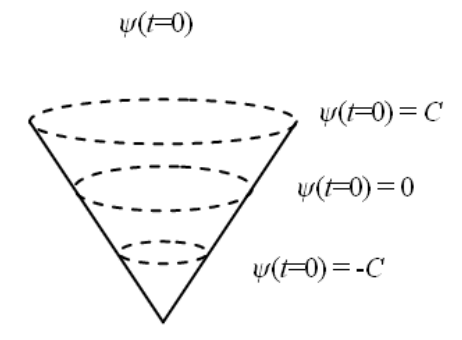

(b)

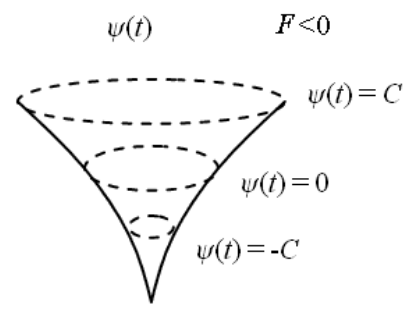

(c)

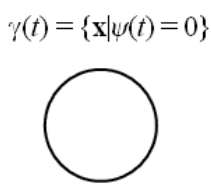

(d)

Figure 1: A level set evolution example. (a) The initial front, (b) the computed level set zero, (c) the updated surface for $F<0$ and (d) the new front stage [34]. 
The speed function plays a central role in level set methods as its value and normal direction determine the propagation velocity and direction of the front interface, as it follows:

$$
F=F_{\text {prop }}+F_{c u r v}+F_{a d v}
$$

where $F_{\text {prop }}$ is the propagation expansion speed, $F_{a d v}$ is the advection speed and $F_{\text {curv }}$ is the curvature speed [2]. This paper deals with the two first terms to model the front propagation discarding the $F_{a d v}$ component as part of the proposed speed function. We define a new $F_{\text {prop }}$ component, as described in the next section, maintaining $F_{\text {curv }}$ as:

$$
F_{\text {curv }}=-\varepsilon K
$$

where $\varepsilon$ is a constant and $K$ is the curvature:

$$
K=\left(\frac{\psi_{x x} \psi_{y}^{2}-2 \psi_{y} \psi_{x} \psi_{x y}+\psi_{y y} \psi_{x}^{2}}{\left(\psi_{x}^{2} \psi_{y}^{2}\right)^{3 / 2}}\right)
$$

$F$ may be dependent on: (i) local properties are determined in each front point, by local geometric information such as curvature; (ii) global properties are based on shape and front position; (iii) independent properties are based on external features such as pixel intensity, texture or statistical information [2].

For image segmentation a numerical solution for the level set equations needs a discrete grid domain in addition to computational techniques for speed function extension, such as global extension and narrow-band extension [35]. All formalism for level set implementation is presented in [34].

\section{Target detection driven by speckle statis- tics}

Targets in SAR images are heterogeneous regions on a homogeneous background. In this section the target detection problem is replaced by heterogeneity detection. Thus, we propose a propagation model with positive velocity in homogeneous regions of the SAR image and negative velocity in heterogeneous regions. This propagation model includes a homogeneity 
measure, provided by a local and adaptive statistical analysis, as it was mentioned earlier in [20] for SAR image filtering purpose. This section provides details of the proposed method for detecting targets, followed by description of the methods used to assess the segmentation results.

\subsection{The Propagation speed model}

Let $\mathbf{x}$ be a pixel in a SAR image $Y,(\mathbf{x} \in Y)$. According to [20] a measure of homogeneity in a region of a SAR image can be estimated in a window $W_{M}$ of $M \mathrm{x} M$ pixels, in terms of the standard deviation to mean ratio as stated in Eq.3. The adaptive scheme consists in changing the window size $M$, automatically, while the pixel neighborhood is heterogeneous. This decision is done by comparing the $\sigma_{z}$ value with a threshold $T$. The ideal value of $T$ is $\beta_{z}=0.5227 / \sqrt{L}$ which corresponds to a homogeneous area.

This scheme regards a subset of the elements in the current window, i.e. only the samples along the boundary of it participate in decision making about the size of the next window, improving the time complexity. As expected, the estimated homogeneity of the terrain reflectivity is more accurate for larger windows due to the number of boundary samples. In fact, smaller windows imply less accurate results[20].

Thus, $T$ is a function of $M$ and it is given by:

$$
T=1+\frac{\sqrt{1+2 \beta_{z}^{2}}}{\sqrt{2 M^{2}}} \beta_{z} .
$$

When applied to all $\mathbf{x} \in Y$ the proposed methodology follows the steps:

1. The window size $M$ is initialized with a maximum value;

2. Estimate the parameters $T$ and $\sigma_{z}$ by using Eq. 3 and Eq. 14 respectively, in a neighborhood window $W_{M}$;

3. if $\sigma_{z}>T$, then $W_{M}$ is a heterogeneous region, $M$ decreases and the algorithm returns to step 2, else $W_{M}$ is homogeneous and the propagation velocity is adjusted by:

$$
F_{\text {prop }}=T-\sigma_{z}
$$

4. return to step 1 . 
The proposed $F_{\text {prop }}$ speed component (Eq.15) presents $F_{\text {prop }}<0$ for heterogeneous and $F_{\text {prop }}>0$ for homogeneous regions. Moreover, this relationship is independent of the window size $M$ which is initialized with large values and does not change $F_{\text {prop }}$ in heterogeneous areas.

Thus, the proposed velocity model for target detection in SAR images is:

$$
F=(-\varepsilon K)+\left(T-\sigma_{z}\right)
$$

Fig. 2 illustrates the processing steps of the proposed algorithm applied to synthetic images, which were artificially contaminated with 8-looks speckle statistics. Fig. 2(a) depicts the original image and Fig. 2(b) is its contaminated version. Fig. 2(c) shows the $F_{\text {prop }}$ matrix computed according to Eq. 9. This image represents the motion pattern of the surface $(\psi(t))$. Furthermore, the fronts $(\gamma(t))$ evolve by following this motion pattern. Inspired in [18] we have also used this initial set of fronts to start the method. Fig. 2(d) is an intermediary stage of the front propagation and Fig. 2(e) is the final result image. It can be observed in the test images that in the interior of targets (where $F_{\text {prop }}<0$ ) the level set propagation decreases, while it increases in the background. This results in a modified version of the initial front before the level set computation, one which is estimated by an adaptive windowing scheme.

\subsection{Segmentation Assessment}

We have assessed the proposed approach and compared it with straightforward algorithms as the one introduced by Huang et. al [2] within the level set framework, Iterated Conditional Modes [36] under the Ising model and entropic thresholding [31] segmentation methods applied to synthetic and real SAR images. These segmentation methods, available as MATLAB scripts, were implemented to evaluate fine details and target preservation ability in comparison with the proposed one.

1. The level set framework for oil slick segmentation in SAR images, developed by Huang et. al [2], computes the image intensity gradient and the curvature flow to track object (slick) boundaries in the scene. The speed in which the front propagates as states Eq. 11 is governed by $F_{\text {prop }}$ which is derived by the intensity gradient and $F_{\text {curv }}$ by the curvature flow. The front propagation of an oil slick edge concerning $F_{\text {prop }}$ is given by 


$$
\begin{aligned}
& I_{\text {average }}=\left(I_{\text {lower }}+I_{\text {high }}\right) / 2 \\
& F_{\text {prop }}= \begin{cases}I(x, y)-I_{\text {lower }} \text { if } I(x, y) \leq I_{\text {average }} \\
I_{\text {high }}-I(x, y) & \text { if } I(x, y) \geq I_{\text {average }}\end{cases}
\end{aligned}
$$

where $I(x, y)$ denotes the intensity of a pixel at the position of $x, y$, while $I_{\text {lower }}$ and $I_{\text {high }}$ denote the minimum and maximum threshold of an oil slick, respectively.

This intensity model allows the level surface to move towards the pixels in the source image with values between $I_{\text {lower }}$ and $I_{\text {high }}[2]$.

2. ICM algorithm

The value of a pixel in an image is highly dependent on the values of neighboring pixels specially in high resolution imagery. image is simply random noise. Markov random fields (MRF) are defined in terms of conditional probabilities associated with spatial neighborhoods. Thus, a bayesian framework is adopted and the a priori global distribution is chosen as Gibbs distribution.

Image segmentation based on Markov Random fields (MRF) model [37] estimates configuration of labels $X=\left(X_{1}, \ldots X_{M N}\right)$ to form a pixel realization $Y=\left(Y_{1}, \ldots Y_{M N}\right)$, where the image specifies the gray levels for all pixels in an $M x N$ lattice. The ICM is proposed by

\section{Experimental Results}

In the following experiments, we track targets in noisy images and show that the proposed approach presents superior visual results when compared with well-known methods as entropic thresholding and ICM. In our algorithm, the level set propagation velocity is driven by a homogeneity grade and the method discards the use of empirical parameters. However, it is necessary to initialize the maximum neighborhood size $(M)$ and other parameters as the number of looks $(L)$ to establish speckle statistics and the level set parameters $\Delta t$ and $\varepsilon$. Aiming at minimizing the computational cost, we adopted the following parameters to all the tests: the initial window size is $M=15, \Delta t=$ 0.5 and $\varepsilon=0.1$. These parameters were chosen according to numerical restrictions and aspects drawn in [34]. The $L$ parameter is dependent on the 
SAR image generation process, while the adopted initial level set is according to $[18]$.

\subsection{Simulated Images}

Circumventing difficulties on segmenting high intensity noise images (e.g. 1look amplitude images) for target detection purpose, the experiments using simulated speckled images accomplished the target detection task as accurately as possible. These tests validated the propagation model according to a reference image (non noisy version). The front propagation indicated that the method succeeded in locating and enclosing targets and that it is independent of the noise intensity.

\subsection{Real Images}

We have assessed the proposed approach and compared it with straightforward algorithms as thresholding [31] and Iterated Conditional Modes [36] segmentation methods applied to real single-look and multilook SAR images. We aimed at evaluating fine details and target preservation ability of all these methods in real noisy images. The performance of the algorithm was also compared with the level set framework introduced by Huang et. al [2].

The segmentation results were obtained by using a real SAR image for target detection in the ocean. Fig. 4(a) displays a RADARSAT-1 image of 3-looks, ScanSAR Wide mode, of an area in the Southwestern South Atlantic with ships in training. Fig. 4(b) and Fig. 4(c) present the results provided by the thresholding and ICM methods, respectively. We can observe that there is still remaining segmentation noise in the processed images using the other methods except for the proposed one. 


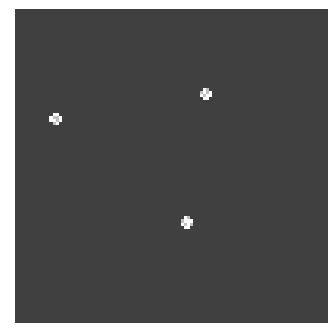

(a)

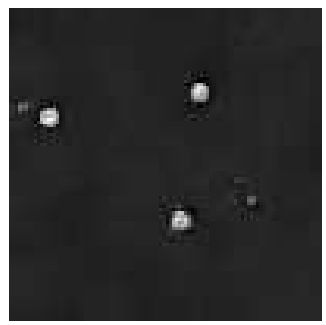

(c)

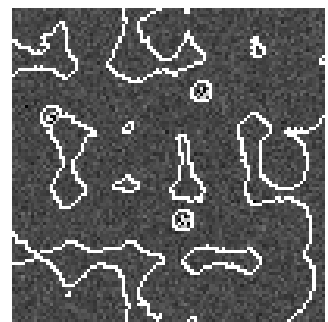

(e)

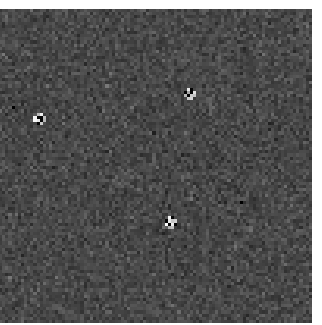

(b)

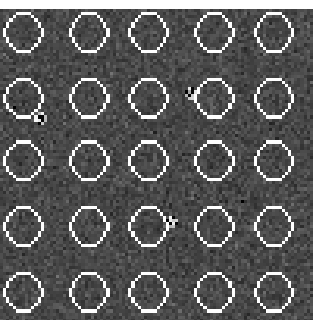

(d)

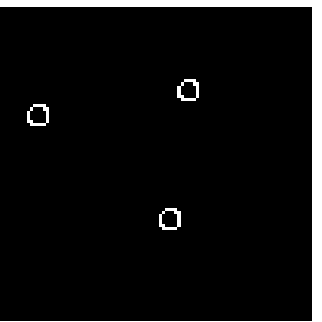

(f)

Figure 2: Level set evolution driven by speckle statistics: (a) A synthetic image, (b) its noisy version with 8-looks speckle statistics, (c) $F_{\text {prop }}$ distribution, (d) random initial level set, (e) intermediary stage and (f) the final result. 


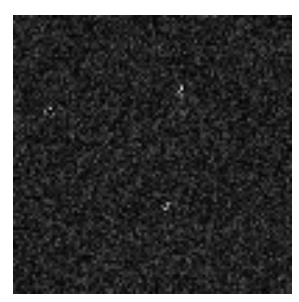

(a)

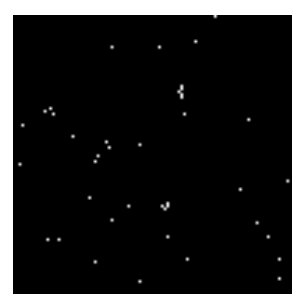

(b)

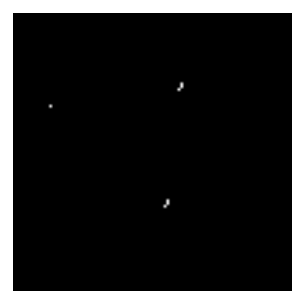

(c)

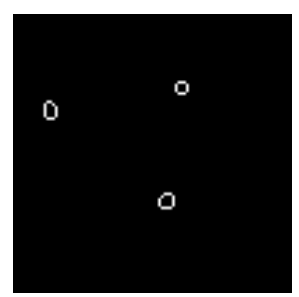

(d)

Figure 3: Segmentation results for different algorithms applied to a simulated SAR image. (a) Image with 1-looks speckle statistics, (b) thresholding result, (c) Huang method result and (d) the proposed method result. 


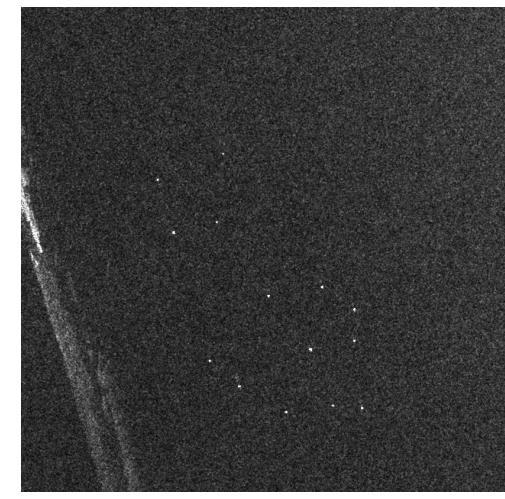

(a)

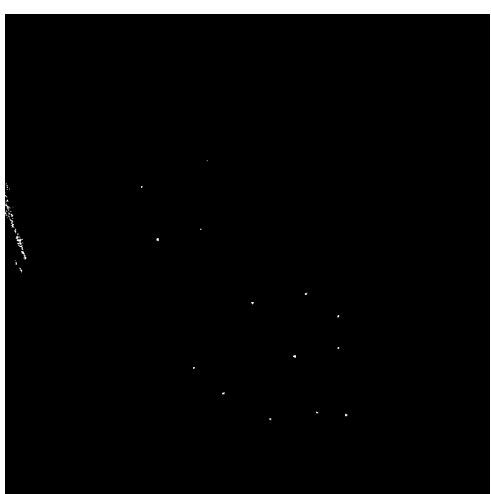

(c)

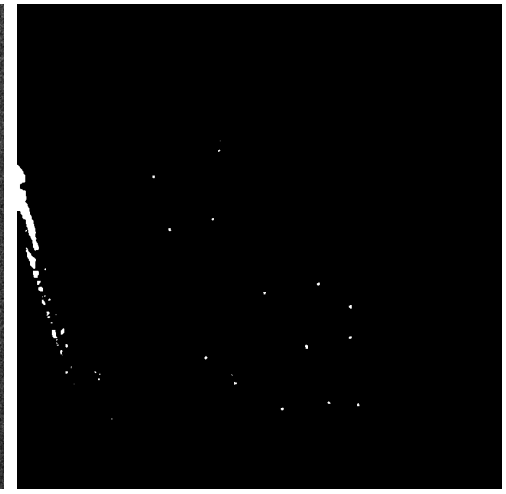

(b)

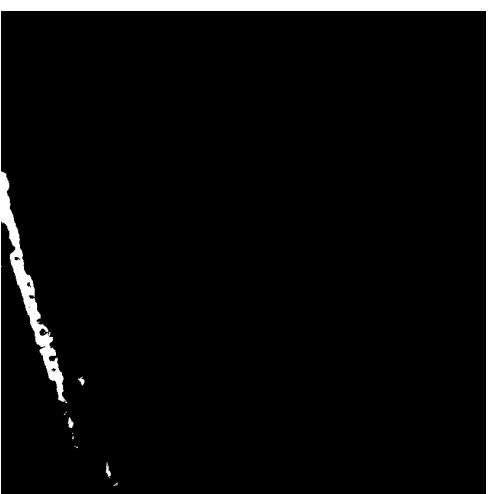

(d)

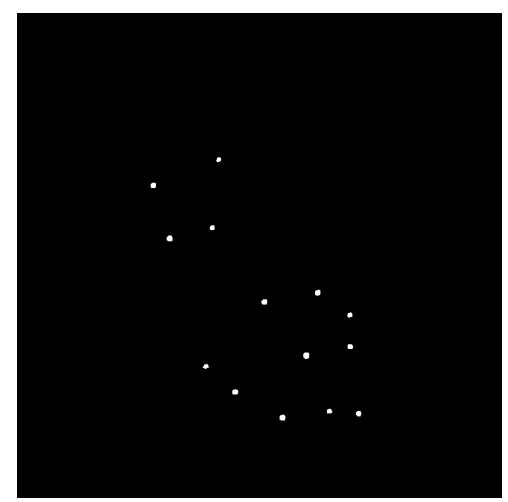

(e)

Figure 4: Segmentation results for different algorithms applied to a real SAR image. (a) Original 3-looks SAR image (820 x 820 pixels) containing ships, (b)the thresholding result, (c) the ICM result, (d) Huang method result and (e) the proposed method result. 


\section{Conclusions}

In this paper we have proposed a novel approach for target detection in amplitude SAR images, based on level set methods and incorporating speckle noise statistics in the algorithm design. One advantage of this method is the fact that it requires neither preprocessing nor postprocessing to track targets in noisy images. When applied to real and simulated speckled images this approach presented low sensitivity to this type of multiplicative noise. Furthermore, this robust method worked independently of target size and traditional speckle filtering. Preliminary results were promising, conveying illustrations on synthetic and real images, compared with other algorithms. Experiments were performed on synthetic and real SAR images contaminated by speckle noise following the Rayleigh and square root of gamma distributions. Further developments must include faster versions of the segmentation algorithms, such as a narrow band level set approach or an initial step based on Fast Marching methods.

\section{Acknowledgments}

The authors are grateful to $\mathrm{CNPq}$ for the financial support. We also would like to thank Renato Feijó for providing the RADARSAT images and Alejandro Frery for synthetic ones.

\section{References}

[1] A. C. Frery, A. H. Correia, and C. C. Freitas. Classifying multifrequency fully polarimetric imagery with multiple sources of statistical evidence and contextual information. IEEE Trans. on Geoscience and Remote Sensing, 45(10):3098-3109, October 2007.

[2] B. Huang, H. Li, and X. Huang. A level set method for oil slick segmentation in sar images. Int. Journal of Remote Sensing, 26(6):1145-1156, March 2005.

[3] A.C. Frery; N.D.A. Mascarenhas;. Sar image filtering with the icm algorithm. International Geoscience and Remote Sensing Symposium, 4:2185 - 2187, Aug 1994. 
[4] G. Moser and S.B. Serpico. Generalized minimum-error thresholding for unsupervised change detection from sar amplitude imagery. IEEE International Geoscience and Remote Sensing, 44(10):2972-2982, October 2006.

[5] Q. Jiang, E. Aitnouri, S. Wang, and D. Ziou. Automtic detection for ship target in sar imagery using pnn-model. Canadian Journal of Remote Sensing, 26(4):297-305, August 2000.

[6] Mariví Tello, Carlos López-Martínez, and Jordi J. Mallorqui. A novel algorithm for ship detection in sar imagery based on wavelet transform. IEEE Geoscience and Remote Sensing Letters, 2(2):201-205, April 2005.

[7] R. C. P. Marques, E. A. Carvalho, R. C. S. Costa, and F. N. S. Medeiros. Filtering effects on sar images segmentation. Lecture Notes in Computer Science, 3124:1041-1046, 2004.

[8] A. Niedermeier, E. Romaneessen, and S. Lehner. Detection of linear features in sar images: Application to the road network extraction. IEEE Trans. Geoscience and Remote Sensing, 38(5):2270-2281, September 2000 .

[9] O. Germain and P. Réfrégier. Statistical active grid for segmentation refinement. Pattern Recognition Letters, 22:1125-1132, 2001.

[10] P. Moreels and S. E. Smrekar. Watershed identification of polygonal patterns in noisy sar images. IEEE Trans. on Image Processing, 12(7):740750, July 2003.

[11] P. Martin, P. Réfrégier, F. Goudail, and F. Guérault. Influence of the noise model on level set active contour segmentation. IEEE Trans. on Pattern Analysis and Machine intelligence, 26(6):799-803, June 2004.

[12] Ushizima D M, Calado R T, and Rizzatti E G. Leukocyte detection using nucleus contour propagation. Lecture Notes in Computer Science, 4091:389-396, aug 2006. MIAR 2006.

[13] T. Kadir and M. Brady. Unsupervised non-parametric region segmentation using level sets. In Proc. of IEEE international Conference on Computer Vision, volume 2, pages 1267-1274, October 2003. 
[14] Jean-Francois Aujol, Gilles Aubert, and Laure Blanc-Féraud. Waveletbased level set evolution for classification of texture images. IEEE Trans. on Image Processing, 12(12):1634-1641, December 2003.

[15] N. Paragios, O. Mellina-Gottardo, and V. Ramesh. Gradient vector flow fast geometric active contours. IEEE Trans. Pattern Analysis and Machine intelligence, 26(3):402-407, March 2004.

[16] M. S. Allili and D. Ziou. An automatic segmentation combining mixture analysis and adaptive region information: A level set approach. In Proc. of Canadian Conference on Computer and Robot Vision, pages 73-80, May 2005.

[17] J. Kim, J. W. Fisher, A. Yezzi, M. Cetin, and A. S. Willsky. A nonparametric statistical method for image segmentation using information theory and curve evolution. IEEE Trans. on Image Processing, 14(10):1486-1502, October 2005.

[18] I. B. Ayed, A. Mitiche, and Z. Belhadj. Multiregion level-set partitioning of synthetic aperture radar images. IEEE Trans. on Pattern Analysis and Machine intelligence, 27(5):793-800, May 2005.

[19] J. S. Lee. Speckle analysis and smoothing of synthetic aperture radar images. Computer Graphics and Image Processing, 17:24-32, 1981.

[20] J. M. Park, W. J. Song, and W. A. Pearlman. Speckle filtering of sar images based on adaptive windowing. IEE Proceedings Vision, Image and Signal Processing, 146(33):191-197, 1999.

[21] J. C. Curlander and R. N. McDonough. Synthetic Aperture Radar: Systems and Signal Processing. John Wiley \& Sons, New York, April 1991.

[22] A. Achim, P. Tsakalides, and A. Bezerianos. Title: Statistical characterisation and modelling of sar images. Signal Processing, 82(1):69-92, Jan 2002.

[23] G.D. De Grandi, J.S. Lee, and D.L. Schuler. Target detection and texture segmentation in polarimetric sar images using a wavelet frame: Theoretical aspects. 45(11):3437-3453, November 2007. 
[24] F. N. S. Medeiros, N. D. A. Mascarenhas, and L. F. Costa. Evaluation of speckle noise map filtering algorithms applied to sar images. Int. Journal of Remote Sensing, 24(24):5197-5218, December 2003.

[25] C. C. F. Yanasse, A. C. Frery, and S.J. S. Sant'Anna. Stochastic distributions and the multiplicative model: relations, properties, estimators and applications to sar image analysis. Technical Report INPE-5630NTC/318, INPE, São José dos Campos, SP, Brazil, 1995.

[26] A. C. Frery, H.-J. Mller, C. C. Freitas, and S.J.S. Sant'Anna. A model for extremely heterogeneous clutter. IEEE Trans. on Geoscience and Remote Sensing, 35(3):648-659, May 1997.

[27] A. C. Frery. Algumas Ferramentas Estatísticas na Síntese, Processamento e Análise de Imagens de Radar de Abertura Sintética. Phd thesis, INPE, São José dos Campos, São Paulo, Brazil, 1993. (In portuguese).

[28] P.R. Vieira. Maximum likelihood and icm image classifiers. Master's thesis, INPE, São José dos Campos, São Paulo, Brazil, 1996. (In portuguese).

[29] F. Cribari-Neto, A. C. Frery, and M. F. Silva. Improved estimation of clutter properties in speckled imagery. Comput. Stat. Data Analysis, 40(8):801-824, October 2002.

[30] Agus Zainal Arifin and Akira Asano. Image segmentation by histogram thresholding using hierarchical cluster analysis. Pattern Recognition Letters, 2006. Article in Press.

[31] W. K. Pratt. Digital Image Processing. John Wiley \& Sons, New York, 1991.

[32] P. Wang, J. Chong, and H. Wang. Ship detection of the airborne sar images. In IEEE International Geoscience and Remote Sensing Symposium (IGARSS00), volume 1, page 348350, 2000.

[33] I. Dydenko, F. Jamal, O. Bernard, J. Hooge, I. E. Magnin, and D. Friboulet. A level set framework with a shape and motion prior for segmentation and region tracking in echocardiography. Medical Image Analysis, 10:162-177, 2006. 
[34] J. A. Sethian. Level Set Methods and Fast Marching Methos: Evolving Interfaces in Computational Geometry, Fluid Mechanics, Computer Vision and Materials Science. Cambridge University Press, Cambridge, 1998.

[35] R. Malladi, J. Sethian, and B. C. Vemuri. Shape modeling with front propagation: A level set approach. IEEE Trans. on Pattern Analysis and Machine intelligence, 17(2):158-175, February 1995.

[36] Charles W. Therrien. Decision Estimation and Classification: An introduction to Pattern Recognition and Related Topics. John Wiley \& Sons, New York, February 1989.

[37] J. Besag. On the statistical analysis of dirty pictures. Journal of the Royal Statistical Society, 48(3):259-302, 1986. 